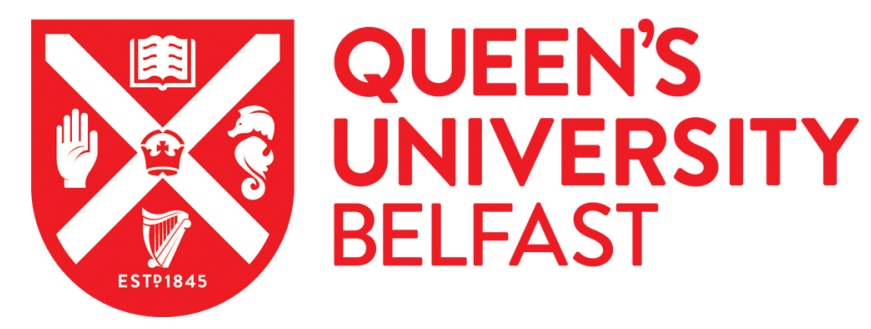

\title{
Cooperative OFDM-IM Relay Networks with Partial Relay Selection under Imperfect CSI
}

Crawford, J., \& Ko, Y. (2018). Cooperative OFDM-IM Relay Networks with Partial Relay Selection under Imperfect CSI. IEEE Transactions on Vehicular Technology. https://doi.org/10.1109/TVT.2018.2853672

\author{
Published in: \\ IEEE Transactions on Vehicular Technology
}

Document Version:

Peer reviewed version

Queen's University Belfast - Research Portal:

Link to publication record in Queen's University Belfast Research Portal

Publisher rights

(c) 2018 IEEE.

This work is made available online in accordance with the publisher's policies. Please refer to any applicable terms of use of the publisher.

\section{General rights}

Copyright for the publications made accessible via the Queen's University Belfast Research Portal is retained by the author(s) and / or other copyright owners and it is a condition of accessing these publications that users recognise and abide by the legal requirements associated with these rights.

Take down policy

The Research Portal is Queen's institutional repository that provides access to Queen's research output. Every effort has been made to ensure that content in the Research Portal does not infringe any person's rights, or applicable UK laws. If you discover content in the Research Portal that you believe breaches copyright or violates any law, please contact openaccess@qub.ac.uk. 


\title{
Cooperative OFDM-IM Relay Networks with Partial Relay Selection under Imperfect CSI
}

\author{
James Crawford, Student Member, IEEE, and Youngwook Ko, Senior Member, IEEE
}

\begin{abstract}
In this work, we investigate the performance of cooperative orthogonal frequency division multiplexing with index modulation (OFDM-IM) with the low complexity greedy detection (GD). In particular, we propose a novel partial relay selection (PRS) scheme whose search criteria are designed to exploit the IM sub-carriers. To provide low-complexity receiver, we further examine the energy-sensing based GD design for the cooperative OFDM-IM. For the performance analysis we derive novel upper bound and approximate closed form solutions for both the average index error probability (IEP) and the average symbol error probability (SEP) over Nakagami- $m$ fading channels with imperfect channel state information (CSI) at the relays and destination. Unlike the information theoretical works, in presence of positive detection error in the relays, the derived expressions provide a useful insight into the error performance of cooperative OFDM-IM under various fading conditions. The numerical and simulation results clearly present that the proposed scheme harmonizing partially selected relays and their IM sub-carriers with GD can outperform the benchmark schemes, under uncertain CSI, at reduced complexity.
\end{abstract}

Index Terms-OFDM-IM, index modulation, cooperative diversity, energy detection.

\section{INTRODUCTION}

Index modulation (IM) is a range of promising new modulation techniques which have increased in popularity in recent years. Due to its ability to transmit data without the consumption of additional bandwidth or no additional power, $\mathrm{IM}$ is a promising candidate for 5G [1]. Moreover, index modulation is an ideal solution for other applications that fall under the $5 \mathrm{G}$ umbrella, such as machine type communications (MTC) due to its flexibility in terms of data-rate, complexity, and energy efficiency (EE).

Inspired by the concept of spatial modulation (SM) [2], subcarrier index modulation (SIM) for OFDM [3] was developed. Here, the indices of sub-carriers are used as an additional degree of freedom. However, the original SIM was limited in terms of the spectral efficiency (SE). Subsequently, this was rectified with the introduction of OFDM-IM [4]. Since then research interest in OFDM-IM has increased significantly. Some of the major contributions can be found in [5]-[13]. However, there has been less of a focus on OFDM-IM for MTC systems, where energy efficiency and system complexity is critical. To address this issue, a low-complexity energy based detection scheme for OFDM-IM was introduced in [14].

Copyright (c) 2015 IEEE. Personal use of this material is permitted. However, permission to use this material for any other purposes must be obtained from the IEEE by sending a request to pubs-permissions@ieee.org.

The authors are with the Electronics, Communications and Information Technology Institute, Queen's University Belfast, Belfast BT3 9DT United Kingdom (e-mail: \{jcrawford23, y.ko\}@qub.ac.uk).
In addition, the performance of OFDM-IM for in-vehicular D2D wireless networks was analyzed in [15], and [16]. The limitation of the achievable power gain of the energy based detection scheme was improved by utilizing various diversity combining techniques in [17].

Relay networks are an efficient method used to improve the reliability of a communication network. In [18], an overview of relay selection for mutli-carrier systems is presented. In [19], multicarrier relay selection schemes for OFDM are presented. However, to the best of our knowledge partial relay selection (PRS) for index modulated multicarrier systems has not yet been investigated. For PRS, channel information is only required locally at the source node, which enables to achieve high cooperative diversity at reduced overall complexity. Decode-and-forward (DF) relays are the ideal choice for cooperative MTC due to its EE [20], simple interference cancellation [21], and unlike AF relaying does not suffer from high peak-to-average power ratio (PAPR) [22].

In this work, we consider an OFDM-IM based multiple DF relay network, where a source device intends to communicate with a destination device via multiple relays and direct links, under imperfect CSI. For each transmission, we propose the cooperative OFDM-IM with greedy detection (GD) leveraging benefits of designing a novel partial relay selection (PRS) scheme based on OFDM-IM sub-carriers. The main contributions can be summarized as follows:

- We create a novel partial relay selection rule which refers to the IM sub-carriers and ensures cooperative OFDM-IM transmission to improve the reliability. This structure will benefit from exploiting both active subcarriers and relays. We develop GD-based OFDM-IM algorithms with multiple relays to improve the reliability at low complexity.

- For the performance evaluation, we derive approximate and upper bound closed form expressions for both the average index error probability (IEP) and the average symbol error probability (SEP) over Nakagami- $m$ fading channels with imperfect CSI.

- With presence of positive detection errors in relays and destination, the derived expressions provide a useful insight into the error performance of PRS based cooperative OFDM-IM as well as the impact of PRS and relay strategy, under various fading conditions.

- Theoretical guide lines of the performance of the proposed scheme is provided through asymptotic analysis. In particular, using extreme value theory, we examine various asymptotic cases: ultra-dense relay networks and large degree of imperfect channel estimation. 
- The numerical results clearly show that the proposed scheme is superior to the benchmark schemes, in terms of reliability at a reduced complexity.

\section{SySTEM MODEL}

\section{A. Cooperative OFDM-IM}

Consider an OFDM-IM based multiple DF relay network where a source node $(S)$ intends to communicate to the destination $(D)$, while cooperating with $Q$ DF relays $\left(R_{q}\right)$, for $q=\{1, \ldots, Q\}$ as illustrated in Fig. 1. Assume that the transmissions between $S \rightarrow R_{q}, S \rightarrow D$, and $R_{q} \rightarrow D$, occur in two orthogonal hops. In the first hop the transmissions between $S \rightarrow R_{q}$ and $S \rightarrow D$ occur. In the second hop the transmission between $R_{q} \rightarrow D$ occurs. For each transmission link, we employ a multi-carrier framework with $N_{c}=G N$ total sub-carriers, being divided into $G$ clusters of $N$ subcarriers. Each cluster independently employs the OFDM-IM such that only $K$ of $N$ sub-carriers per cluster are dynamically activated and the information bits are conveyed by both the $K$ active sub-carriers and their active indices. We will examine opportunities of harmonizing best relay selection and dynamic active sub-carriers (which will be discussed in Section II.B).

Specifically as for OFDM-IM in each cluster, set of $K$ active sub-carrier indices represents the index modulation (IM) symbol, denoted by $\mathbf{J}_{g}=\left[i_{g}(1), \ldots, i_{g}(K)\right]$, for cluster $g$, where $i_{g}(k) \in\{(g-1) N+1, \ldots, g N\}$, $g=1, \ldots, G$, and $k=1, \ldots, K$. Correspondingly, a cluster of $K$ data symbols are denoted as $\mathbf{s}_{g}=\left[s_{g}(1), \ldots, s_{g}(K)\right]$ where $s_{g}(k) \in \mathcal{S}$ is a $M$-ary symbol and $\mathcal{S}$ denotes the complex symbol constellation. Each OFDM-IM symbol per cluster is generated using both $\mathbf{J}_{g}$ and $\mathbf{s}_{g}$, as $\mathbf{x}_{g}=$ $\left[x_{g}((g-1) N+1), x_{g}((g-1) N+2), \cdots, x_{g}(g N)\right]^{T}$, where $x_{g}(\alpha) \in \mathcal{S}$, for $\alpha \in \mathbf{J}_{g}$, and $x_{g}(\widetilde{\alpha})=0$ for $\widetilde{\alpha} \notin \mathbf{J}_{g}$. The transmit power of non-zero data symbols is defined as $\mathbb{E}\left|x_{g}(\alpha)\right|^{2}=E_{S} / \tau$, where $E_{s}$ is the average power per $M$-ary symbol, and $\tau=K / N$ is the sub-carrier sparsity ratio. Subsequently, $G$ clusters are concatenated together to create the OFDM-IM block, i.e., $\mathbf{x}=\left[\mathbf{x}_{1}^{T}, \mathbf{x}_{2}^{T}, \cdots, \mathbf{x}_{G}^{T}\right]^{T}$.

The number of potential IM symbols is considered to be $2^{\left\lfloor\log _{2}\left(\begin{array}{c}N \\ K\end{array}\right)\right\rfloor}$ for simplicity and efficient mapping of data bits. Hence, in each cluster, $m_{J, g}=\left\lfloor\log _{2}\left(\begin{array}{c}N \\ K\end{array}\right)\right\rfloor$ bits are used to modulate the active sub-carrier indices (i.e., IM symbols) and $m_{S, g}=K \log _{2} M$ bits determine the $M$-ary symbols to modulate the sub-carriers of the active indices.

Based on these, in the first hop, the received signals at $R_{q}$ and $D$ in the frequency domain can be given as,

$$
\begin{gathered}
\mathbf{y}_{S R_{q}}=\mathbf{H}_{S R_{q}} \mathbf{x}+\mathbf{n}_{S R_{q}}, \\
\mathbf{y}_{S D}=\mathbf{H}_{S D} \mathbf{x}+\mathbf{n}_{S D},
\end{gathered}
$$

respectively, where $\mathbf{H}_{\Sigma}$ is the channel matrix for $\Sigma \in$ $\left\{S R_{q}, S D\right\}, \quad \mathbf{x}$ is an $N \times 1$ OFDM-IM block, $\mathbf{n}=$ $\left[n(1), \ldots, n\left(N_{c}\right)\right]^{T}$ is an independent additive complex Gaussian noise vector, i.e., $\mathbf{n} \sim \mathcal{C N}\left(\mathbf{0}, N_{0} \mathbf{I}\right)$, where $\mathbf{I}$ is the identity matrix and $N_{0}$ is noise variance, and the average transmit SNR per active sub-carrier is defined as $\rho=E_{S} /\left(N_{0} \tau\right)$.

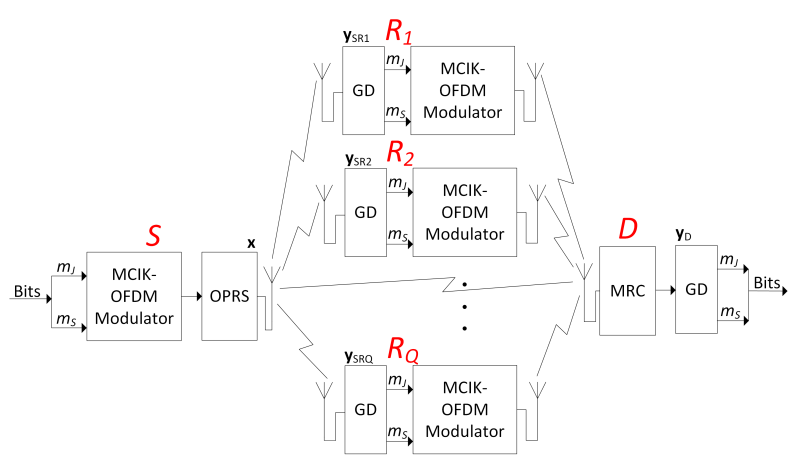

Fig. 1. Block diagram of cooperative OFDM-IM network with a single source, single destination, and multiple relays. Using the channel information between $S \rightarrow R$, the best relay is selected on a per sub-carrier basis.

In the second hop the signals are received via $R_{q} \rightarrow D$, which is given as,

$$
\mathbf{y}_{R_{q} D}=\mathbf{H}_{R_{q} D} \widetilde{\mathbf{x}}+\mathbf{n}_{R_{q} D}
$$

where $\mathbf{H}_{R_{q} D}$ is the channel matrix in the frequency domain between $R_{q} \rightarrow D, \widetilde{\mathbf{x}}$ is the $N \times 1$ OFDM-IM block that was recovered at $R_{q}$. Subsequently $\mathbf{y}_{S D}$ and $\mathbf{y}_{R_{q} D}$ are combined at $D$ using the maximal ratio combiner (MRC). Omitting any subscripts of node for clarification, the received signal at any node can be given as $y(j)=h(j) x(j)+n(j)$ for sub-carrier $j$, where $j=(1, \ldots, N)$.

For simplicity in analysis and without loss of generality, we focus on only one cluster and the cluster index $g$ is omitted from subscripts hereafter as each cluster operates independently. The partial relay selection process with imperfect CSI will be addressed in detail in the following.

\section{B. Opportunistic Partial Relay Selection (OPRS) in Coopera- tive OFDM-IM}

In practical systems, notice that CSI may not be estimated perfectly at the node. We assume that the estimated CSI between any two nodes, in the frequency domain, is given as, $\hat{\mathbf{H}}=\mathbf{H}-\mathbf{E}$, where $\mathbf{H}=\operatorname{diag}\left(h(1), \ldots, h\left(N_{c}\right)\right)$ is the channel matrix, $h(\alpha)$ is assumed to face independent, and identically distributed (i.i.d.) Nakagami- $m$ fading distribution for $\alpha \in \mathbf{J}, m \geq 0.5, \mathbf{E}=\operatorname{diag}\left(e(1), \ldots, e\left(N_{c}\right)\right)$ is the channel estimation error matrix, $e(\alpha) \sim \mathcal{C N}\left(0, \nu_{e}\right)$, and $\nu_{e}$ is the variance of the channel estimation errors, representing the level of CSI uncertainty. $\hat{\mathbf{H}}=\operatorname{diag}\left(\hat{h}(1), \ldots, \hat{h}\left(N_{c}\right)\right)$ can accordingly consist of $\hat{h}(\alpha)=h(\alpha)-e(\alpha), \forall \alpha$.

We now propose the opportunistic partial relay selection (OPRS) rule for cooperative OFDM-IM networks. Suppose that $\hat{\mathbf{H}}_{S R}=\left[\hat{\mathbf{H}}_{S R_{1}}, \ldots, \hat{\mathbf{H}}_{S R_{Q}}\right]^{T}$ is known at $S$, where $\hat{\mathbf{H}}_{S R_{q}}=\operatorname{diag}\left(\hat{h}_{S R_{q}}(1), \ldots, \hat{h}_{S R_{q}}\left(N_{c}\right)\right)$. Particularly, OPRS refers to the CSI only during the first hop and chooses the best relay(s) that provides the largest channel gain, for each active sub-carrier, between $S$ and $R_{q}$. 
The criterion for the relay selection at the $\alpha^{\text {th }}$ active subcarrier is to find the best among $Q$ relays, whose channel gain satisfies,

$$
\hat{h}_{S \tilde{r}_{\alpha}}(\alpha)=\max _{q \in\{1, \ldots, Q\}}\left|\hat{h}_{S R_{q}}(\alpha)\right|^{2},
$$

where the subscript $\tilde{r}_{\alpha}$ denotes the selected relay. Notice that the selected relay $\tilde{r}_{\alpha}$ forwards signals on each active subcarrier $\alpha$ and this post-selection relaying process allows at most $K$ relays to be chosen. Hence, let $\mathcal{R}=\left(\tilde{r}_{1}, \ldots, \tilde{r}_{K}\right)$ denote a set of selected relays, where $|\mathcal{R}| \leq K$.

The instantaneous SNR of links $S \rightarrow \tilde{r}_{\alpha_{2}} S \rightarrow D$, and $\tilde{r}_{\alpha} \rightarrow D$, is given as $\gamma_{\Delta}(\alpha)=\rho\left|\hat{h}_{\Delta}(\alpha)\right|^{2} /\left(\rho \nu_{e}+1\right)=$ $\bar{\gamma}\left|\hat{h}_{\Delta}(\alpha)\right|^{2}$, where $\Delta \in\left\{S \tilde{r}_{\alpha}, S D, \tilde{r}_{\alpha} D\right\}$ and $\bar{\gamma}$ denotes the average SNR per sub-carrier. Applying the MRC to the received signals from both $\tilde{r}_{\alpha}$ and $D$, the instantaneous SNR is defined as, $\gamma_{D}(\alpha)=\bar{\gamma}\left(\left|\hat{h}_{S D}(\alpha)\right|^{2}+\left|\hat{h}_{\tilde{r}_{\alpha} D}(\alpha)\right|^{2}\right)=$ $\bar{\gamma}\left|\hat{h}_{D}(\alpha)\right|^{2}$, where $\hat{h}_{D}(\alpha)$ is the estimated channel gain at the output of the MRC at $D$.

Subsequently, the received signals at $\tilde{r}_{\alpha}$ and $D$ are demodulated using the greedy detector (GD). In the following section the GD algorithm for OPRS-OFDM-IM is given.

\section{Greedy Detector (GD) Receiver}

The GD requires a two stage detection process, where the indices of the active sub-carriers and the corresponding $M$ ary symbols are estimated separately. In the first stage, GD measures the signal energy of each sub-carrier $|y(j)|^{2}$, and detects $K$ active sub-carriers with the greatest energy, which is given as, $\hat{\alpha}=\arg \max _{j}|y(j)|^{2}$. Note that channel estimation errors have no effect on energy detection.

In the second stage, the non-zero $M$-ary symbols in $\mathrm{x}$ are detected, applying the maximum likelihood (ML) decision individually to the $K$ estimated active sub-carriers, which is given as, $z(\hat{\alpha})=\arg \min _{x(\hat{\alpha}) \in \mathcal{S}}\|y(\hat{\alpha})-\hat{h}(\hat{\alpha}) x(\hat{\alpha})\|^{2}$, where $\hat{h}(\hat{\alpha})$ is the $\alpha^{\text {th }}$ diagonal element of $\hat{\mathbf{H}}$. Thus, referring to $(\hat{\alpha}, z(\hat{\alpha}))$, we can detect both IM and $M$-ary symbols.

\section{ERror Probability ANALYSis}

\section{A. Index Error Probability (IEP) Analysis}

1) Instantaneous IEP for OPRS-OFDM-IM: The IEP is defined as the case when the greatest noise energy of any inactive sub-carrier is greater than the energy of any active sub-carrier, i.e., $I E P=\max _{\widetilde{\alpha}}|n(\widetilde{\alpha})|^{2}>|h(\alpha) x(\alpha)+n(\alpha)|^{2}$. This can occur at $D$ under two different scenarios. In the first scenario an error occurs at $D$ when the $S \rightarrow R$ transmission is received correctly and the transmission at $D$ is received incorrectly, i.e., $P_{1}=\left[1-I E P_{S \tilde{r}_{\alpha}}\right] \times I E P_{D}$, where $\left[1-I E P_{S \tilde{r}_{\alpha}}\right]$ is the complementary IEP at $R$, and $I E P_{D}$ is the IEP at $D$. In the second scenario the $S \rightarrow R$ transmission is received incorrectly, i.e., $P_{2}=I E P_{S \tilde{r}_{\alpha}}$, where $I E P_{S \tilde{r}_{\alpha}}$ is the IEP at $R$. Hence, the overall instantaneous IEP is determined by a miss-detection of sub-carrier indices. Using the law of the total probability, this can be represented as, $P_{I}=K / N \sum_{\alpha=1}^{N} P_{1}+P_{2}$.

The approximate instantaneous IEP at $\Lambda \in\left\{S \tilde{r}_{\alpha}, D\right\}$ is formulated as [15], $I E P_{\Lambda} \approx(N-K) / 2 \cdot \exp \left[-\rho\left|h_{\Lambda}(\alpha)\right|^{2} / 2\right]$.

Substituting $I E P_{\Lambda}$ into $P_{I}$, gives the instantaneous IEP , which is given as,

$$
\begin{aligned}
P_{I} & \approx \frac{K}{N} \sum_{\alpha=1}^{N} \frac{N-K}{2}\left\{e^{-\frac{\rho\left|h_{S \tilde{r}_{\alpha}}(\alpha)\right|^{2}}{2}}+e^{-\frac{\rho\left|h_{D}(\alpha)\right|^{2}}{2}}\right. \\
& \left.-\frac{N-K}{2} e^{-\frac{\rho\left(\left|h_{S \tilde{r}_{\alpha}}(\alpha)\right|^{2}+\left|h_{D}(\alpha)\right|^{2}\right)}{2}}\right\} .
\end{aligned}
$$

Remark 1. Note from (7), $P_{I}$ is not affected by $\nu_{e}$. This can be explained by the fact that energy detection does not require channel information. Hence, a poorly estimated channel will have no effect on the IEP.

2) Average IEP for OPRS-OFDM-IM: To examine the average IEP, we take the expectation of $P_{I}$, which is given as,

$$
\begin{aligned}
& \bar{P}_{I}=K \underbrace{\mathbb{E}_{\left|h_{S \tilde{r}_{\alpha}}\right|^{2}}\left\{I E P_{S \tilde{r}_{\alpha}}\right\}}_{I_{1}}+K \underbrace{\mathbb{E}_{\left|h_{D}\right|^{2}}\left\{I E P_{D}\right\}}_{I_{2}} \\
& -K \underbrace{\mathbb{E}_{\left|h_{S \tilde{r}_{\alpha}}\right|^{2}}\left\{I E P_{S \tilde{r}_{\alpha}}\right\}}_{I_{1}} \underbrace{\mathbb{E}_{\left|h_{D}\right|^{2}}\left\{I E P_{D}\right\}}_{I_{2}} .
\end{aligned}
$$

To solve (6), the higher order statistics can be used to derive the PDF of the largest random variable, $\left|h_{S \tilde{r}_{\alpha}}\right|^{2}$, but it becomes mathematically intractable for complex generalized channel fading models, such as Nakagami- $m$ fading. Therefore, the extreme value theory approach can be employed to yield an asymptotic distribution for the maximum of $Q$ i.i.d. RVs. This approach is clarified to provide a tight bound to the exact PDF even for as little as $Q=10$, [23]. Given $\left|h_{S \tilde{r}_{\alpha}}\right|^{2}$ is Gamma distributed the asymptotic distribution of the largest of $Q$ Gamma random variables is given in the form of a Gumbel random variable, i.e., $z=\left(\left|h_{S \tilde{r}_{\alpha}}\right|^{2}-\mu_{Q}\right) / \beta_{Q}$. The generalized inverse and the auxiliary function are given as $\mu_{Q}=W^{-1}(m, Q-1 / Q) / m$, and $\beta_{Q}=$ $\Gamma(m)\left[m Q W^{-1}(m, Q-1 / Q)^{m-1} \exp \left(-W^{-1}(m, Q-1 / Q)\right)\right]^{-1}$, respectively, where $\Gamma(\cdot)$ is the gamma function, and $W(\cdot, \cdot)$ is the regularized lower incomplete Gamma function.

In (6), the solution to $I_{1}$ can be found by taking the expectation of $I E P_{S \tilde{r}_{\alpha}}$ w.r.t. $z=\left(\left|h_{S \tilde{r}_{\alpha}}\right|^{2}-\mu_{Q}\right) / \beta_{Q}$ that is Gumbel distributed, and its PDF is given as, $g_{z}(z)=$ $\exp \left[-z-e^{-z}\right] / \beta_{Q}$. As for $I_{2}$, we take notice that $\left|h_{D}\right|^{2}$ follows Gamma distribution, and its PDF is given as, $f_{\left|h_{D_{\alpha}}\right|^{2}}(h)=m^{2 m} h^{2 m-1} e^{-h m} / \Gamma(2 m)$. The detailed expressions for $I_{1}$ and $I_{2}$ are referred to Appendix A.

Hence, substituting the above closed-form outcomes of $I_{1}$ and $I_{2}$ into (6) yields the average IEP expression:

$$
\bar{P}_{I}=K \frac{N-K}{2}\left[\Psi+\Xi-\frac{N-K}{2} \Psi \Xi\right],
$$

where $\Psi(\rho)=\exp \left(-\rho \mu_{Q} / 2\right) \gamma\left[\rho \beta_{Q} / 2+1, \exp \left(\mu_{Q} / \beta_{Q}\right)\right]$, $\gamma[m, x]=\int_{0}^{x} t^{m-1} e^{t} \mathrm{~d} t$ is the lower incomplete gamma function, and $\Xi(\rho)=[2 m /(2 m+\rho)]^{2 m}$. 
Remark 2. Note from (5), $\bar{P}_{I}$ behaves concavely with $K$ for given $N, \rho$, and $h_{\Lambda}$, due to $\partial^{2} \bar{P}_{I} / \partial K^{2}<0, \forall K$. The concave behaviour of (5) can result in a value for $K$ that minimizes the $\bar{P}_{I}$ as either the smallest or largest over a given set of $K \mathrm{~s}$.

\section{B. Data Symbol Error Probability (SEP) Analysis}

1) Instantaneous SEP for OPRS-OFDM-IM: The SEP is defined as the number of symbols in error divided by the number of symbols transmitted, where we consider errors in both sub-carrier index and data symbol recovery. Referring to the derivation of the instantaneous IEP, the overall instantaneous SEP is formulated as,

$$
P_{s}=\frac{K}{N} \sum_{\alpha=1}^{N} S E P_{S \tilde{r}_{\alpha}}+S E P_{D}-S E P_{S \tilde{r}_{\alpha}} S E P_{D}
$$

where for each sub-carrier, $S E P_{\Lambda}$ denotes the instantaneous SEP at $\Lambda \in\left\{S \tilde{r}_{\alpha}, D\right\}$, which is given as,

$$
S E P_{\Lambda}=I E P_{\Lambda}+P_{M, \Lambda}-\left[I E P_{\Lambda} \times P_{M, \Lambda}\right]
$$

where $P_{M, \Lambda}$ is the instantaneous SEP of $M$-ary symbols. Notice that we use the approximated $P_{M, \Lambda}$ as [24], $P_{M, \Lambda} \approx \exp \left[-3 \bar{\gamma}\left|h_{\Lambda}(\alpha)\right|^{2} / 2 M-2\right] / 6+$ $\exp \left[-4 \bar{\gamma}\left|h_{\Lambda}(\alpha)\right|^{2} / 2 M-2\right] / 2$.

Particularly, substituting $I E P_{\Lambda}$ and $P_{M, \Lambda}$, into $S E P_{\Lambda}$, we have,

$$
\begin{aligned}
S E P_{\Lambda} & \approx \frac{N-K}{2} e^{-\frac{\rho\left|h_{\Lambda}(\alpha)\right|^{2}}{2}}+\frac{1}{6} e^{-\frac{3 \bar{\gamma}\left|h_{\Lambda}(\alpha)\right|^{2}}{2 M-2}} \\
& +\frac{1}{2} e^{-\frac{4 \bar{\gamma}\left|h_{\Lambda}(\alpha)\right|^{2}}{2 M-2}}-\frac{N-K}{4} \\
& \times\left(\frac{e^{\frac{-\rho(M-1)+3 \bar{\gamma}}{2 M-2}\left|h_{\Lambda}(\alpha)\right|^{2}}}{3}-e^{\frac{-\rho(M-1)+4 \bar{\gamma}}{2 M-2}\left|h_{\Lambda}(\alpha)\right|^{2}}\right) .
\end{aligned}
$$

Remark 3. For clarity, (10) is the instantaneous SEP for a single hop between any two nodes within the proposed network. Moreover, it is conditioned on the channel of a single sub-carrier. Inserting (10) into (8), the instantaneous SEP can be simply obtained taking into account the index symbols and data symbols for OPRS-OFDM-IM.

2) Average SEP for OPRS-OFDM-IM: Taking the expectation of the instantaneous SEP over the channel gain, the average SEP can be written as:

$$
\begin{aligned}
& \bar{P}_{s}=K \underbrace{\mathbb{E}_{\left|h_{S \tilde{r}_{\alpha}}\right|^{2}}\left\{S E P_{S \tilde{r}_{\alpha}}\right\}}_{I_{3}}+K \underbrace{\mathbb{E}_{\left|h_{D}\right|^{2}}\left\{S E P_{D}\right\}}_{I_{4}}
\end{aligned}
$$

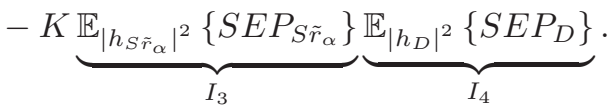

In (11), notice that $I_{3}$ and $I_{4}$ are the expectation of (9). Hence, the expectation of $I_{3}$ (and $I_{4}$ ) can involve the expectations of $I E P_{\Lambda}$ and $P_{M, \Lambda}$. In particular, $I_{3}$ consists of two unique expectations, which can be given as,

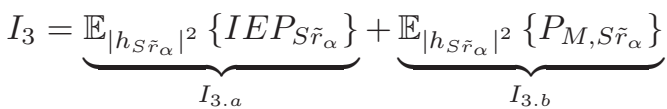

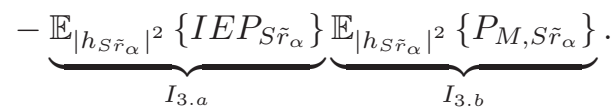

The solution to $I_{3 . a}=I_{1}$, and the solution to $I_{3 . b}$ can be referred to (A.3) and (B.1), respectively.

Using (A.3) and (B.1) and after some mathematical manipulation $I_{3}$ can be expressed as,

$$
\begin{array}{r}
I_{3}=\frac{(N-K) \Psi}{2}+\frac{\Upsilon(3)}{6}+\frac{\Upsilon(4)}{2} \\
-\frac{(N-K) \Psi}{2}\left[\frac{\Upsilon(3)}{6}+\frac{\Upsilon(4)}{2}\right],
\end{array}
$$

where $\Psi$ is recalled to (7), and

$$
\Upsilon(c)=e^{-\frac{c \bar{\gamma} \mu_{Q}}{2 M-2}} \gamma\left(\frac{c \bar{\gamma} \beta_{Q}}{2 M-2}, e^{\frac{\mu_{Q}}{\beta_{Q}}}\right) .
$$

Similarly, to solve $I_{4}$ in (11), we re-express $I_{4}$, referring to $S E P_{\Lambda}$ in (10), as:

$$
\begin{gathered}
I_{4}=\underbrace{\mathbb{E}_{\left|h_{D}\right|^{2}}\left\{I E P_{D}\right\}}_{I_{4 . a}}+\underbrace{\mathbb{E}_{\left|h_{D}\right|^{2}\left\{P_{M, D}\right\}}}_{I_{4 . b}} \\
-\underbrace{\mathbb{E}_{\left|h_{D}\right|^{2}}\left\{I E P_{D}\right\}}_{I_{4 . a}} \underbrace{\mathbb{E}_{\left|h_{D}\right|^{2}\left\{P_{M, D}\right\}} .}_{I_{4 . b}}
\end{gathered}
$$

For brevity, we provide the solution to $I_{4 . a}=I_{2}$, and the solution to $I_{4 . b}$ in (A.5) and (B.2), respectively.

Using these, (15) can be re-written as:

$$
\begin{array}{r}
I_{4}=\frac{(N-K) \Xi}{2}+\frac{\Phi(3 / 2)}{6}+\frac{\Phi(2)}{2} \\
-\frac{(N-K) \Xi}{2}\left[\frac{\Phi(3 / 2)}{6}+\frac{\Phi(2)}{2}\right],
\end{array}
$$

where $\Phi(c)=[c \bar{\gamma} /(M m-m)+1]^{-2 m}$, and $\Xi$ is in (7).

Finally, the average SEP can be found by inserting (13) and (16) into (11). Taking into account the first two distinct terms, we can provide $\bar{P}_{s}$ as,

$$
\begin{aligned}
\bar{P}_{s} & \leq \frac{K}{2}\left\{(N-K) \Psi+\frac{\Upsilon(3)}{3}+\Upsilon(4)-(N-K) \Psi\right. \\
& \times\left[\frac{\Upsilon(3)}{3}+\Upsilon(4)\right]+(N-K) \Xi+\frac{\Phi(3 / 2)}{3} \\
& \left.+\Phi(2)-(N-K) \Xi\left[\frac{\Phi(3 / 2)}{3}+\Phi(2)\right]\right\} .
\end{aligned}
$$

Remark 4. We have now examined the average error probability overall symbols including both index symbols and data symbols on the active sub-carriers. Note that (17) clearly shows that $\bar{P}_{s}$ decreases with $\rho$, through $\Psi, \Xi, \Upsilon(\cdot)$ and $\Phi(\cdot)$, for a given $K, N$, and $m$. 


\section{Special Case: Rayleigh fading}

As a special case, let us consider Rayleigh fading channel coefficient on each sub-carrier. Accordingly, using the Gamma distribution when $m=1$ and taking the expectation of $P_{s}$, we can compute the upper bound average SEP as

$$
\begin{aligned}
\bar{P}_{s . R} & \leq K\left\{\frac{(N-K) \Lambda\left(\frac{\rho}{2}\right)}{2}+\frac{\Lambda\left(\frac{3 \bar{\gamma}}{2 M-2}\right)}{6}+\Lambda\left(\frac{3 \bar{\gamma}}{2 M-2}\right)\right. \\
& -\frac{(N-K) \Lambda\left(\frac{\rho}{2}\right)}{2}\left[\frac{\Lambda\left(\frac{3 \bar{\gamma}}{2 M-2}\right)}{6}+\Lambda\left(\frac{3 \bar{\gamma}}{2 M-2}\right)\right] \\
& +\frac{2(N-K)}{(2+\rho)^{2}}+\frac{2}{3} \Omega(3)+\Omega(2)-\frac{2(N-K)}{(2+\rho)^{2}} \\
& \left.\times\left[\frac{2}{3} \Omega(3)+\Omega(2)\right]\right\},
\end{aligned}
$$

where $\Lambda(c)=(Q+c+1) \cdot \mathrm{B}(c+1, Q+1)$, where $\mathrm{B}(x, y)=\int_{0}^{1} t^{x-1}(1-t)^{y-1} \mathrm{~d} t$ is the Beta function, and $\Omega(c)=[(M-1) /(2 M-2+c \bar{\gamma})]^{2}$. The details of the derivation are shown in Appendix $\mathrm{C}$.

Remark 5. Given $d=-\lim _{\rho \rightarrow \infty} \ln \left(I_{4 . R}\right) / \ln (\rho)=2$, it is shown that the diversity order of $\bar{P}_{s . R}$ is limited to 2 , and for $Q>2$ the error performance will marginally increase.

Remark 6. Note, from (18) the Beta function is the dominant term in $I_{3 . R}$, i.e., the first hop. Hence, as $Q$ increases linearly, $I_{3 . R}$ decreases exponentially for a given $N, K, M$, and $\bar{\gamma}$. However, typical of DF relay schemes, the performance of OPRS-OFDM-IM is determined by the weakest hop.

Remark 7. For a fixed $\nu_{e}, \bar{P}_{s . R}$ becomes a deterministic value as $\bar{\gamma}$ increases. Hence, for a fixed $\nu_{e}$ there is zero diversity order and power gain as there is an error floor. Thus, the error performance does not improve for large SNR.

\section{Asymptotic ANALYSis}

In this section various extreme cases are considered in order to investigate the asymptotic behaviour of OPRS-OFDMIM, in terms of the average SEP. For simplicity in analysis, the upper bound average SEP over Rayleigh fading (18) is considered in asymptotic cases.

\section{A. Large SNR: $\rho \rightarrow \infty$}

Consider a scenario where the SNR grows large. As a special case, perfect CSI is also considered, i.e., $\nu_{e} \rightarrow 0$.

Hence, the overall average SEP in this scenario is given as,

$$
\begin{aligned}
\lim _{\rho \rightarrow \infty} \bar{P}_{s . R} & \approx \frac{(2 \tau)^{Q}}{\overline{S N R}^{Q}}+\frac{(\tau 2 M-2 \tau)^{Q}}{\left(3 \overline{S N R}^{Q}\right.}+\frac{(\tau M-\tau)^{Q}}{(2 \overline{S N R})^{Q}} \\
& =\Theta\left[M^{Q} \tau^{Q} \overline{S N R}^{-Q}+M^{2} \tau^{2} \overline{S N R}^{-2}\right] .
\end{aligned}
$$

where $\Theta[\cdot]$ is big-theta notation, and $\overline{S N R}=E_{s} / N_{0}$.

From (19), it is shown that the achievable diversity order is given as $d=-\lim _{\overline{S N R} \rightarrow \infty} \ln \left(\bar{P}_{s . R}\right) / \ln (\overline{S N R})=2$. Intuitively, this is because the achievable diversity order by the detection at $D$ is dominant, even with the use of $Q$ relays. However, it is clear that increasing $Q$ will reduce the first term in (19). Hence, leading to the enchanced SEP.

\section{B. Large Number of Relay: $Q \rightarrow \infty$}

Consider a large number of relays in a dense D2D network. In this asymptotic case when $Q \rightarrow \infty$ perfect CSI is considered, i.e., $\nu_{e} \rightarrow 0$.

In this extreme scenario the average SEP of the first hop, i.e., $I_{3 . R}$, is given as,

$$
\begin{aligned}
\lim _{Q \rightarrow \infty} I_{3 . R} & \approx(N-K) Q^{-\frac{\rho}{2}} \Gamma\left(\frac{\rho}{2}+1\right)+\frac{Q^{-\frac{3 \rho}{2 M-2}}}{3} \\
& \times \Gamma\left(\frac{3 \rho}{2 M-2}+1\right)+Q^{-\frac{2 \rho}{M-1}} \Gamma\left(\frac{2 \rho}{M-1}+1\right) \\
& =\Theta\left[Q^{-\frac{\overline{S N R}}{M \tau}}\right] .
\end{aligned}
$$

Hence, (20) shows how $I_{3 . R}$ scales with respect to the diversity gain (i.e., $\overline{S N R} / \tau M$ ) if $Q$ is large.

Notice that $I_{4 . R}$ is unaffected by $Q$ as it represents the average SEP between $S \rightarrow D$ where no relays are used. Hence, the overall average SEP in this scenario is given as,

$$
\lim _{Q \rightarrow \infty} \bar{P}_{s . R}=\Theta\left[M^{2} \tau^{2} \overline{S N R}^{-2}\right] .
$$

From (21) it can be seen that the diversity order of 2 is achieved in this scenario. In particular, it is found that the system performance in such extreme case is dominated by the second hop.

\section{Severe CSI Uncertainty: $\nu_{e} \rightarrow 1$}

Now, consider when $\nu_{e} \rightarrow 1$, i.e., the estimated CSI becomes highly unreliable. Here, a large SNR is also consider. In this scenario the average SEP is given as,

$$
\begin{aligned}
\lim _{\nu_{e} \rightarrow 1} \bar{P}_{s . R} & \approx\left(\frac{M-1}{2 M-2}\right)^{Q}+\left(\frac{M-1}{M-1}\right)^{Q} \\
& +\left(\frac{M-1}{2 M+1}\right)^{2}+\left(\frac{M-1}{M+1}\right)^{2} \\
& =\Theta\left[\left(\frac{M-1}{M+1}\right)^{Q}+\left(\frac{M-1}{M+1}\right)^{2}\right] .
\end{aligned}
$$

As can be observed from (22), increasing $Q$ decreases the average SEP, even in the presence of highly uncertain CSI. It is important to mention that in this asymptotic case, the average SEP is not affected by $\overline{S N R}$ and instead, the average SEP will be floored at the error level which relies on $M$ and $Q$, for very large $v_{e} \gg 0$. This observation will be validated by simulations in the next section.

\section{Simulation Results And Discussion}

We now present simulation results for OPRS-OFDM-IM. For illustrations, we consider $N_{c}=128$ total sub-carriers with various values of the parameters, that is, $N \in\{2,4\}, K=$ $1, M \in\{2,4\}, m \in\{0.5,1,2,3\}, Q \in\{1, \ldots, 8,10\}$, and $\nu_{e} \geq 0.003$. Here, the average SEP is evaluated on a per cluster basis, as each cluster of $N$ sub-carriers is detected individually. Minimum mean square error (MMSE) channel estimation is used in various simulations. In this case, the variance of the channel estimation error matrix becomes, $\nu_{e}=1 /(\rho+1)$. 


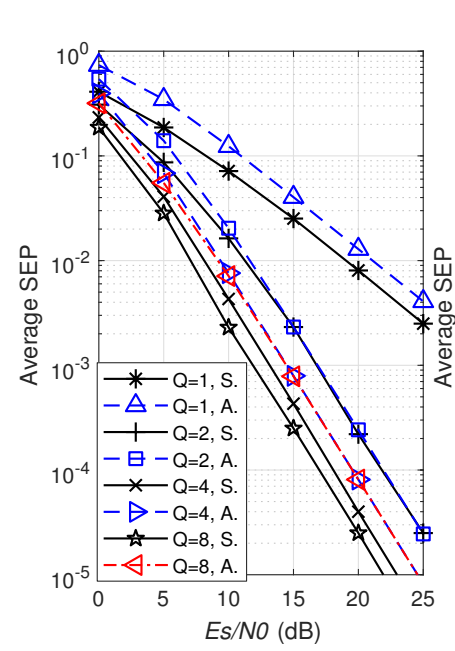

(a)

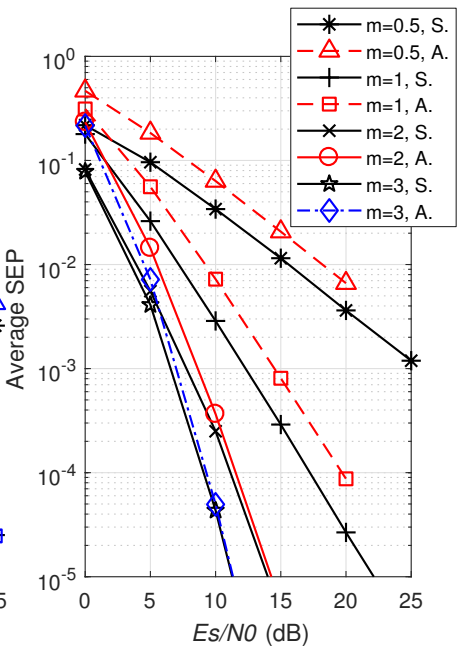

(b)
Fig. 2. Comparison of Analytical (A.) and Simulated (S.) Average SEP of OPRS-OFDM-IM for $N=4, K=1, M=4$, and (a) $m=1$, and $Q=\{1,2,4,8\}$, and (b) $m=\{0.5,1,2,3\}, Q=10$.

Fig. 2.a depicts the comparison between the analytical (A.) and simulated (S.) average SEP of the OPRS-OFDM-IM with GD, when using the MMSE channel estimation with $N=4$, $K=1, M=4, m=1$, and $Q=\{1,2,4,8\}$. It is observed that the average SEP significantly improves as $Q$ increases. For example, the power gain of $12 \mathrm{~dB}$ can be achieved for the average SEP of $10^{-3}$ between $Q=1$ and $Q=2$. However, between $Q=2$ and $Q=4$ the power gain is only marginal, and the diversity order remains the same. This can be explained by the average SEP being dominated by the weakest hop, i.e., the average SEP at $D$. It is also observed from Fig. 2.a that the analytical (A.) curves from (18) provide a tight bound to the simulation results with an approximately $2 \mathrm{~dB}$ gap for the average SEP of $10^{-3}$ when $Q=\{1,8\}$.

Fig.2.b depicts the average SEP with various $m$, i.e., $m=$ $\{0.5,1,2,3\}$, and $Q=10$. It is observed that as $m$ increases the average SEP significantly decreases. In particular, the power gain of $14 \mathrm{~dB}$ is achieved for the average SEP of $10^{-3}$ between $m=0.5$ and $m=1$. The diversity order increases as $m$ in increased, where the diversity order, in the presence of Nakagami- $m$ fading, is given as $d=\arg \min _{\mathcal{D}}(\mathcal{D})$, $\mathcal{D} \in\left\{d_{R}, d_{D}\right\}, d_{R}=Q m$ is the diversity order attained at $R$ and $d_{D}=2 m$ is the diversity order achieved at $D$. In this figure it is depicted with $Q=10$ that the analytical results from (17) provide a tight bound to the simulation, providing less than $1 \mathrm{~dB}$ difference for large $m(\geq 2)$.

Fig. 3 now illustrates the comparison with the proposed OPRS-OFDM-IM with GD, against the benchmark OPRSOFDM with ML, both using MMSE channel estimation, in terms of average SEP, with $N=2, K=1, M=2$, $m=\{0.5,1,2,3\}, Q=\{1, \ldots, 8\}$, and a SNR of (a) 10 $\mathrm{dB}$ and (b) $15 \mathrm{~dB}$. For a fair comparison the transmission data-rate of both schemes are set to be same, i.e., 4 bits per cluster. Fig. 3.a depicts a marginal difference between the proposed scheme and the benchmark scheme in terms of the average SEP, when $m=1,2,3$ and in the presence of

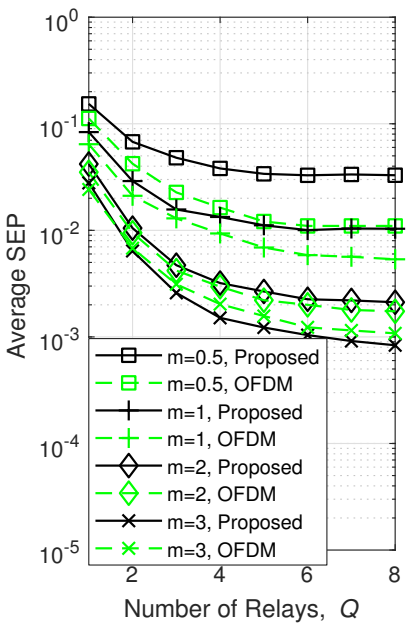

(a)

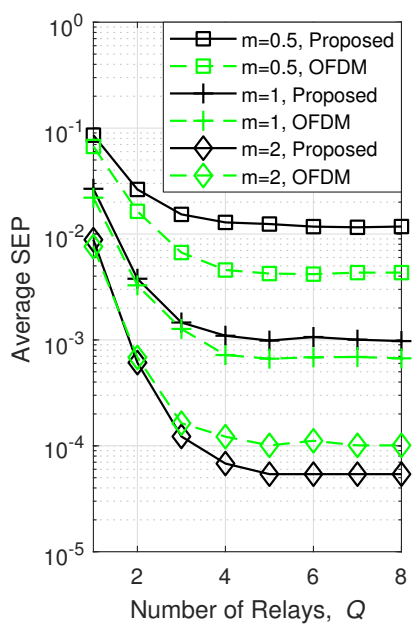

(b)
Fig. 3. Comparison with the proposed OPRS-OFDM-IM and MMSE, over the OPRS-OFDM with ML and MMSE when $N=2, K=1, M=2$, $m=\{0.5,1,2,3\}, Q=\{1, \ldots, 8\}$, and SNR of (a) $10 \mathrm{~dB}$ (b) $15 \mathrm{~dB}$.

channel estimation errors. In particular, it is observed that the proposed scheme outperforms the benchmark when $m=3$. In the presence of channel estimation errors, this is because the proposed scheme does not require channel information for the detection of sub-carriers. However, for $m=0.5$ it can be observed that the benchmark scheme outperforms the proposed scheme. Intuitively, this is caused by the degraded energy detection for sub-carrier indices at $m<1$.

In Fig. 3.b the comparison between the proposed OPRSOFDM-IM and MMSE, against the benchmark OPRS-OFDM with ML and MMSE in terms of average SEP is illustrated for an SNR of $15 \mathrm{~dB}$. Here, it can be seen that the proposed scheme outperforms the benchmark scheme when $m=2$. It is clear that as the SNR increases, the smaller $m$ is needed for the proposed scheme to outperform the benchmark scheme.

Fig. 4 depicts the simulated average SEP of OPRS-OFDMIM using both the GD and ML which are compared with $\nu_{e}=$ $\{0.003,0.007,0.03,0.07,0.3,0.7,1\}, N=4, K=1, M=4$, $m=1$, and $Q=\{1,2,3\}$. It is clear from Fig. 4.a that when $Q=1$, ML outperforms GD, for lower values of $\nu_{e}$. However, as $\nu_{e}$ increases the performance of GD and the ML detector are identical. Also, as $Q$ increases, the performance gain of $\mathrm{ML}$ over GD decreases. In Fig. 4.b the SNR is increased. Here, the performance gain of ML over GD is reduced in comparison with Fig. 4.a, when $Q=1$. For $Q>1$ the performance of GD and ML become identical. It is important to note that, due to large complexity of the ML detector, the GD is the preferable detector for OPRS-OFDM-IM in the presence of channel estimation errors, as it has the same performance as the ML while maintaining a reduced complexity.

Fig. 5.a compares the proposed OPRS-OFDM-IM with GD, against the best relay selection (BRS) based OFDM-IM with GD when $N=2, K=1, M=2, m=1, Q=4$, and $\nu_{e}=$ $\{0.5,0.1,0.05\}$, where the BRS chooses the relay with the highest end-to-end SNR at each sub-carrier. It can be seen that, over a range of CSI uncertainty, proposed scheme outperforms the BRS scheme for $Q=4$. In particular as $\nu_{e}$ decreases the 


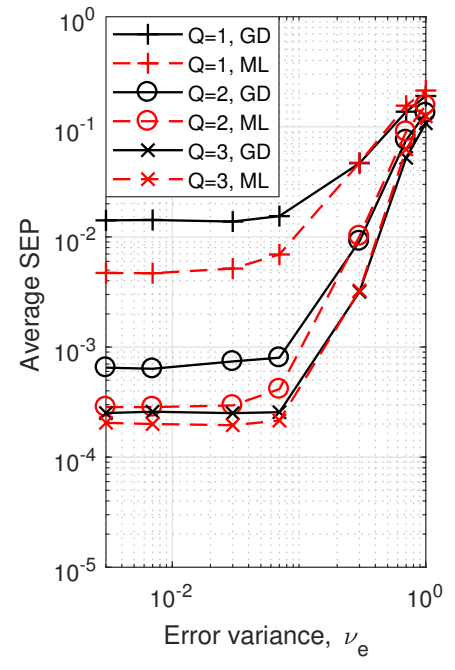

(a)

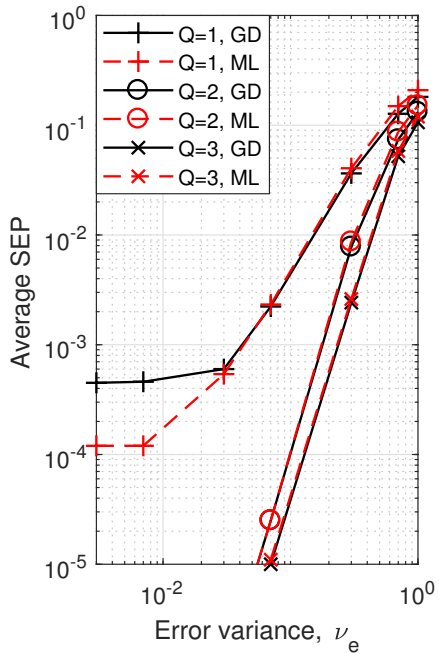

(b)
Fig. 4. Using the OPRS-OFDM-IM, the proposed GD is compared over the ML, with $\nu_{e}=\{0.003,0.007,0.03,0.07,0.3,0.7,1\}, N=4, K=1$, $M=4, m=1, Q=\{1,2,3\}$, and SNR of (a) $15 \mathrm{~dB}$ (b) $30 \mathrm{~dB}$.

performance improvement increases at high SNRs.

In Fig. 5.b we further illustrate the comparison against BRS-OFDM-IM with GD, in terms of $\nu_{e}=0.5$ and various $Q=\{2,4,6,8\}$. It is clear from the figure that the proposed scheme outperforms the BRS scheme as $Q \geq 2$ increases. In particular, the error floor of the proposed scheme with $Q=4$ is lower than that of the BRS scheme with $Q=8$. This comparison clearly presents that the proposed scheme is much less susceptible to channel uncertainty than the BRS scheme.

\section{CONCLusions}

We proposed the cooperative OFDM-IM with GD for dual hop multiple DF relays network, designing a novel PRS scheme based on OFDM-IM sub-carriers. The derived expressions provide a useful insight into the error performance of the cooperative OFDM-IM system under various fading conditions. In the presence of detection errors in the relays, we clearly showed that the achievable diversity order is less than or equal to $2 m$, while the power gain is proportional to the number of relays and active sub-carrier index rates. Through asymptotic and simulation results, we have clearly shown that: OPRS-OFDM-IM with channel estimation errors outperforms the benchmark scheme, i.e., OPRS-OFDM, as channel information is not require for the detection of subcarriers; The GD is the preferable detector for OPRS-OFDMIM in the presence of channel estimation errors.

\section{APPENDIX A}

\section{DERIVATION OF $I_{1}$ AND $I_{2}$}

Firstly, $\left|h_{S \tilde{r}_{\alpha}}\right|^{2}=z \beta_{Q}+\mu_{Q}$ and $\left|h_{S \tilde{r}_{\alpha}}\right|^{2}=\beta_{Q} \mathrm{~d} z$ are substituted into $I_{1}$ from (6),

$$
I_{1}=\frac{(N-K) e^{-\frac{\rho \mu_{Q}}{2}}}{2} \int_{-\frac{\mu_{Q}}{\beta_{Q}}}^{\infty} e^{-\left(\frac{\rho \beta_{Q}}{2}+1\right) z-e^{-z}} \mathrm{~d} z
$$

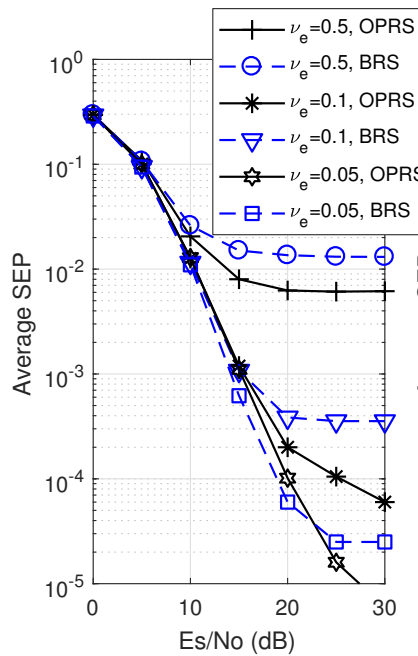

(a)

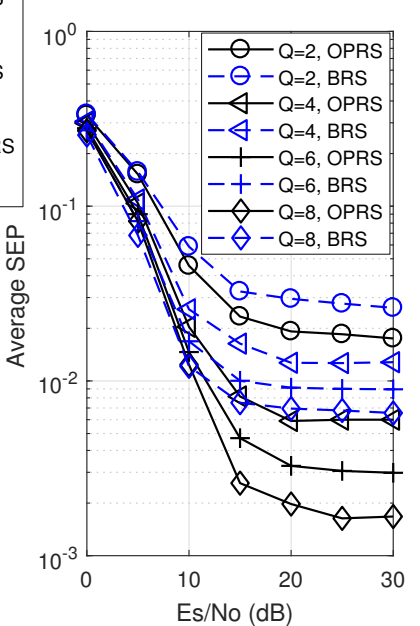

(b)
Fig. 5. Comparison of the proposed scheme, over the BRS-OFDM-IM with $\mathrm{GD}$, in terms of average SEP, with $N=2, K=1, M=2, m=1$, and (a) $\nu_{e}=\{0.05,0.1,0.5\}$, and $Q=4$ (b) $\nu_{e}=0.5$ and $Q=\{2,4,6,8\}$.

From (A.1), an additional substitution, $z=t-\frac{\mu_{Q}}{\beta_{Q}}$ is made, which yields,

$$
I_{1}=\frac{(N-K) e^{\frac{\mu_{Q}}{\beta Q}}}{2} \int_{0}^{\infty} e^{-\left(\frac{\rho \beta_{Q}}{2}+1\right) t-e^{\frac{\mu_{Q}}{\beta_{Q}}} e^{-t}} \mathrm{~d} t .
$$

It is observed that (A.2) is in the form, $\int_{0}^{\infty} \exp \left(-A x-B e^{-x}\right) d x$. The integral solution can be found in [25, eq. (3.331-1)], in the form, $B^{-A} \gamma(A, B)$ conditioned on $[\operatorname{Re} A>0]$. Hence (A.2) becomes,

$$
I_{1}=\frac{(N-K) e^{-\frac{\rho \mu_{Q}}{2}}}{2} \gamma\left(\frac{\rho \beta_{Q}}{2}+1, e^{\frac{\mu_{Q}}{\beta}}\right) .
$$

Secondly, $I_{2}$ is given as,

$$
I_{2}=\frac{N-K}{2} \frac{m^{2 m}}{\Gamma(2 m)} \int_{0}^{\infty} h^{2 m-1} e^{-\left(\frac{\rho}{2}+m\right) h} \mathrm{~d} h .
$$

Referring to [25, (3.381-4)], (A.4) is in the form, $\int_{0}^{\infty} x^{\nu-1} e^{-\mu x} d x$. Hence, the integral solution can be found in [25, eq. (3.381-4)], in the form, $\mu^{-\nu} \Gamma(\nu)$. Therefore,

$$
I_{2}=\frac{N-K}{2}\left(\frac{2 m}{2 m+\rho}\right)^{2 m} \text {. }
$$

\section{APPENDIX B}

\section{DERIVATION OF $I_{3 . b}$ AND $I_{4 . b}$}

Similar to (A.2), $I_{3 . b}$ is in the form, $\int_{0}^{\infty} \exp \left(-A x-B e^{-x}\right) d x$. Hence its solution becomes,

$$
\begin{aligned}
I_{3 . b} & =\frac{e^{-\frac{3 \bar{\gamma} \mu_{Q}}{2 M-2}}}{6} \gamma\left(\frac{3 \bar{\gamma} \beta_{Q}}{2 M-2}, e^{\frac{\mu_{Q}}{\beta} Q}\right) \\
& +\frac{e^{-\frac{4 \bar{\gamma}_{Q}}{2 M-2}}}{2} \gamma\left(\frac{4 \bar{\gamma} \beta_{Q}}{2 M-2}, e^{\frac{\mu_{Q}}{\beta}}\right)
\end{aligned}
$$

Secondly, $I_{4 . b}$ is found to be in the same form as (A.4), i.e., $\int_{0}^{\infty} x^{\nu-1} e^{-\mu x} d x$. Referring to [25, (3.381-4)], $I_{4 . b}$ yields,

$I_{4 . b}=\frac{1}{6}\left(\frac{3 \bar{\gamma}}{2 m(M-1)}+1\right)^{-2 m}+\frac{1}{2}\left(\frac{2 \bar{\gamma}}{m(M-1)}+1\right)^{-2 m}$ 


\section{APPENDIX C \\ DERIVATION OF $I_{3 . R}$ AND $I_{4 . R}$}

Using the higher order statistics, we have $\left|h_{S \tilde{r}_{\alpha}}\right|^{2}$ as the maximum of $Q$ exponential RVs, whose PDF is given as,

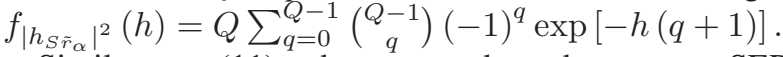

Similar to (11), the upper bound average SEP over Rayleigh fading channel can be given as, $\bar{P}_{s . R}=$ $K\left(I_{3 . R}+I_{4 . R}-I_{3 . R} I_{4 . R}\right)$.

Firstly, $I_{3 . R}$ is formulated in the same manor as, (12). However, it is now found by taking the expectation w.r.t. $\left|h_{S \tilde{r}_{\alpha}}\right|^{2}$, which is formulated as, $I_{3 . R}=I_{3 . a . R}+I_{3 . b . R}-I_{3 . a . R} I_{3 . b . R}$.

Where $I_{3 . a . R}$ is given as,

$$
I_{3 . a . R}=\frac{N-K}{2} Q \sum_{q=0}^{Q-1}\left(\begin{array}{c}
Q-1 \\
q
\end{array}\right)(-1)^{q} \int_{0}^{\infty} e^{-h\left(q+1+\frac{\rho}{2}\right)} \mathrm{d} h .
$$

The integral in (C.1) is in the form, $\int_{0}^{\infty} e^{-p x} d x$. Utilizing integral solution [25, (3.310)], which is in the form, $1 / p$, yields,

$$
I_{3 . a . R}=\frac{(N-K) \mathrm{B}\left(Q+1, \frac{\rho}{2}+1\right)}{2\left(Q+\frac{\rho}{2}+1\right)^{-1}} .
$$

$I_{3 . b . R}$ is formulated in the same manor as, (15). However, as before, it is now found by taking the expectation w.r.t. the Rayleigh channel gain. As before the integral in is in the form, $\int_{0}^{\infty} e^{-p x} d x$. Hence, $I_{3 . b . R}$ becomes,

$$
I_{3 . b . R}=\frac{\mathrm{B}\left(Q+1, \frac{3 \bar{\gamma}}{2 M-2}+1\right)}{6\left(Q+\frac{3 \bar{\gamma}}{2 M-2}+1\right)^{-1}}+\frac{\mathrm{B}\left(Q+1, \frac{2 \bar{\gamma}}{M-1}+1\right)}{\left(Q+\frac{2 \bar{\gamma}}{M-1}+1\right)^{-1}} .
$$

Secondly, $I_{4 . R}$ is simply solved by setting $m=1$ in $I_{4}$.

\section{REFERENCES}

[1] E. Basar, "Index modulation techniques for 5G wireless networks," IEEE Commun. Mag., vol. 54, no. 7, pp. 168-175, Jul 2016.

[2] R. Y. Mesleh, H. Haas, S. Sinanovic, C. W. Ahn, and S. Yun, "Spatial modulation," IEEE Trans. Veh. Technol., vol. 57, no. 4, pp. 2228-2241, Jul. 2008.

[3] R. Abu-alhiga and H. Haas, "Subcarrier-index modulation OFDM," Proc. IEEE PIMRC, Tokyo, Japan, Sep. 2009, pp. 177-181.

[4] E. Basar, U. Aygolu, E. Panayirci, and H. V. Poor, "Orthogonal frequency division multiplexing with index modulation," IEEE Trans. Signal Process., vol. 61, no. 22, pp. 5536-5549, Nov. 2013.

[5] Y. Ko, "A tight upper bound on bit error rate of joint OFDM and multicarrier index keying," IEEE Commun. Lett., vol. 18, no. 10, pp. 17631766, Oct. 2014.

[6] E. Basar, "OFDM with index modulation using coordinate interleaving," IEEE Wireless Commun. Lett., vol. 4, no. 4, pp. 381-384, Aug. 2015.

[7] _ - "On multiple-input multiple-Output OFDM with index modulation for next generation wireless networks," IEEE Trans. Signal Process., vol. 64, no. 15, pp. 3868-3878, Aug. 2016.

[8] M. Wen, E. Basar, Q. Li, B. Zheng, and M. Zhang, "Multiple-mode orthogonal frequency division multiplexing with index modulation," IEEE Trans. Commun., May. 2017.

[9] T. V. Luong and Y. Ko, "A tight bound on BER of MCIK-OFDM with greedy detection and imperfect CSI," IEEE Commun. Lett., Aug. 2017.

[10] - "Impact of CSI uncertainty on MCIK-OFDM: tight, closed-form symbol error probability analysis," IEEE Trans. Veh. Technol., Sep. 2017.

[11] E. Chatziantoniou, Y. Ko, and J. Choi, "Non-orthogonal multiple access with multi-carrier index keying," Proc. Int. Conf. EWC, Dresden, Germany, Aug. 2017, pp. 205-209.
[12] J. Choi and Y. Ko, "TCM for OFDM-IM," IEEE Wireless Commun. Lett., Sep. 2017.

[13] J. Choi, "Coded OFDM-IM With transmit diversity," IEEE Trans. Commun., vol. 65, no. 7, pp. 3164-3171, Jul. 2017.

[14] J. Crawford and Y. Ko, "Low complexity greedy detection method with generalized multicarrier index keying OFDM," Proc. IEEE PIMRC, Hong Kong, China, Aug. 2015, pp. 688-693.

[15] E. Chatziantoniou, J. Crawford, and Y. Ko, "Performance analysis of a low complexity detector for MCIK-OFDM over TWDP fading," IEEE Commun. Lett., vol. 20, pp. 1251-1254, Jun. 2016.

[16] T. V. Luong and Y. Ko, "Symbol error outage performance analysis of MCIK-OFDM over complex TWDP fading," Proc. Int. Conf. EWC, Dresden, Germany, Aug. 2017, pp. 268-272.

[17] J. Crawford, E. Chatziantoniou, and Y. Ko, "On The SEP Analysis of OFDM Index Modulation with Hybrid Low Complexity Greedy Detection and Diversity Reception," IEEE Trans. Veh. Technol., 2017.

[18] P. Guo, Y. Bai, M. Ma, S. Wu, and S. Dang, "Relay technology for multi-carrier systems: a research overview," Proc. IEEE C3IT), Hooghly, India, Feb. 2015, pp. 1-5.

[19] S. Dang, J. Coon, and D. E. Simmons, "Combined bulk/per-subcarrier relay selection in two-hop OFDM systems," Proc. IEEE VTC Spring, Nanjing, China, May 2016, pp. 1-5.

[20] S. Biswas and A. Chandra, "DF versus AF: energy consumption comparison for IEEE 802.15.4 networks," Proc. Int. Conf. COMSNETS, Bangalore, India, Feb. 2014, pp. 1-7.

[21] R. T. L. Rolny, "Future mobile communication from cooperative cells to the post-cellular relay carpet," Ph.D dissertation, Dept. Inform. Technol. and Elect. Eng., ETH-Zürich, Zürich, 2016.

[22] K. Ishibashi and H. Ochiai, "Analysis of instantaneous power distributions for non-regenerative and regenerative relaying signals," IEEE Trans. Wireless Commun., vol. 11, no. 1, pp. 258-265, Jan. 2012.

[23] P. Embrechts, C. Kluppelberg, and T. Mikosch, Modelling extremal events. Springer-Verlag, 1996.

[24] H. A. Suraweera and J. Armstrong, "Simple and accurate approximation to the SEP of rectangular QAM in arbitrary nakagami-m fading channels," IEEE Commun. Lett., vol. 11, no. 5, pp. 426-428, May 2007.

[25] I. Gradshteyn and I. Ryzhik, Table of Integrals, Series, and Products, A. Jeffrey and D. Zwillinger, Eds. Academic Press, 2007.

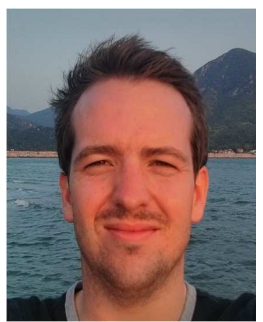

James Crawford received the M.Eng. and $\mathrm{PhD}$ degrees in electrical and electronic engineering from Queen's University, Belfast, U.K., in 2014 and 2018, respectively. After his $\mathrm{PhD}$ he works at Ericsson, Stockholm, Sweden, as a developer. His research interests include multicarrier index keying, and low power device type communications, for the next generation wireless communications.

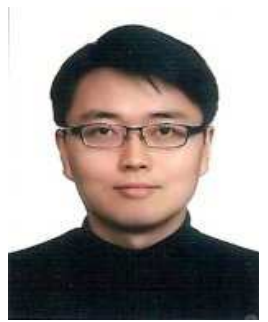

Youngwook Ko received Ph.D. in 2006 and M.S in 2002, in Electrical Engineering from Arizona State University, Tempe, AZ USA, and B.S.E. in Information and Communications Engineering from Hannam University, South Korea. After his Ph.D., he worked at Samsung for two years, as a senior researcher. In 2008, he was in Electrical and Computer Engineering at the University of Alberta, Canada. Between 2010-2013 he was with the CCSR/5GiC, University of Surrey, UK, as a senior research fellow. Since 2013, Dr. Ko works in the ECIT Institute at the Queen's University of Belfast as a Lecturer in Wireless Communications Innovation Centre. He is on the Editorial Board of the Elsevier Journal on Physical Communications and a member of the EPSRC Peer Review Associate College. His research is in the areas of multicarrier index keying, machine type communications, wireless security and vehicular communications. 